\section{Provision of AEDs may not improve survival after sudden cardiac arrest at home}

When sudden cardiac arrest occurs at home, timely medical attention from emergency services can be difficult to achieve. A multicenter clinical trial has been performed to determine whether home use of an automated external defibrillator (AED), before the arrival of emergency medical services, can improve survival in such circumstances.

The patients enrolled in this trial had previously had anterior-wall myocardial infarction but were not eligible to receive an implantable cardioverter-defibrillator. Of the 7,001 patients included, 3,495 were randomly assigned an AED to be used by their companions if a sudden cardiac arrest occurred at home, before calling emergency medical services.

The companions of 167 patients assigned an AED were unable or unwilling to use the device during follow-up (median 37.3 months). The number of patients who died (222 [6.4\%] patients provided with an AED and 228 [6.5\%] controls) and the mechanism of death (e.g. cardiac tachyarrhythmia, heart failure) did not differ between the two patient groups. Home defibrillators were used on 32 patients in the AED group and shock was advised for 13 of these individuals. No inappropriate shock was recorded. Eight patients each in the AED and control groups were successfully resuscitated at home following sudden cardiac arrest.

Access to a home AED did not improve survival in this group of patients with previous anterior-wall myocardial infarction, but the authors of this study attribute this result to a very low event rate, a high proportion of unwitnessed events, and underuse of the AEDs in emergencies, rather than problems with device efficacy.

Original article Bardy GH et al. for the HAT investigators (2008) Home use of automated external defibrillators for sudden cardiac arrest. N Engl J Med 358: 1793-1804

\section{All patients should receive high-dose atorvastatin after CABG surgery}

Patients who have undergone CABG surgery are at particularly high risk of atherosclerosis progression, yet these individuals tend not to receive adequate lipid-lowering therapy. Shah et al.'s post-hoc analysis of patients enrolled in the randomized, double-blind Treating to New Targets (TNT) trial has revealed that high-dose atorvastatin after CABG surgery reduces the incidence of cardiovascular events by $27 \%$, and the need for repeat revascularization by $30 \%$, when compared with a low-dose regimen.

The TNT trial enrolled patients aged 3575 years with coronary heart disease. Exclusion criteria included nephrotic syndrome, liver disease, uncontrolled hypertension or diabetes, and hypersensitivity to statins. Of the 4,654 participants who reported previous CABG surgery, 2,338 were randomly assigned to receive low-dose atorvastatin $10 \mathrm{mg}$ per day and 2,316 to the high-dose regimen $80 \mathrm{mg}$ per day. Highdose atorvastatin was associated with a $51 \%$ reduction in LDL cholesterol from baseline, compared with a $38 \%$ reduction with lowdose atorvastatin. After a median follow-up of 4.9 years, the incidence of major cardiovascular events was significantly lower in the high-dose group than in the low-dose group (hazard ratio $0.73,95 \% \mathrm{Cl} 0.62-0.87 ; P=0.0004)$ as was the need for repeat revascularization (hazard ratio $0.70,95 \% \mathrm{Cl} 0.60-0.82 ; P<0.0001$ ). High-dose atorvastatin was generally well tolerated, but was associated with a trend towards an increased incidence of treatment-related adverse events $(P=0.04)$. The authors recommend that atorvastatin $80 \mathrm{mg}$ per day should be given to all patients who have undergone CABG surgery.

Original article Shah SJ et al. (2008) Intensive lipid-lowering with atorvastatin for secondary prevention in patients after coronary artery bypass surgery. J Am Coll Cardiol 51: 1938-1943 